\title{
Omisión del debido procedimiento y prescripción en la Dirección Regional de Salud, Amazonas, 2017 - 2018
}

\section{Omission of due procedure and prescription in the Regional Health Directorate, Amazonas, 2017 - 2018}

\author{
Janine Giovanna Carrión Ramos ${ }^{1}$, Sonia Tejada Muñoz ${ }^{2}$, Roberto Guevara Aranda ${ }^{3}$
}

\section{RESUMEN}

La investigación se realizó con el objetivo de determinar la omisión del debido procedimiento administrativo que genera la prescripción en los procedimientos administrativos disciplinarios (PAD) en la Dirección Regional de Salud (DIRESA) Amazonas, Perú, 2017 y 2018. El universo muestral fue 57 resoluciones directorales que resuelven la prescripción de los PAD. La metodología de la investigación fue de tipo básico, diseño descriptivo, correlacional, retrospectivo y transversal. El instrumento utilizado fue la ficha de cotejo. Se demostró que la mayor omisión al 100\% fueron dos garantías del debido procedimiento administrativo, que son: El derecho a la presunción de licitud y el cumplimiento de los plazos razonablemente. Así mismo la prescripción del 98\% de los PAD se produjeron en el primer momento para el inicio del PAD. Entonces se concluye que la omisión del debido procedimiento por parte de las autoridades administrativas si generó la prescripción de los procedimientos administrativos disciplinarios.

Palabras claves: Debido procedimiento, prescripción, procedimiento disciplinario

\begin{abstract}
This research was conducted with the objective of determining the omission of due administrative procedure that generates the prescription in disciplinary administrative procedures (PAD) in the Regional Health Directorate (DIRESA) Amazonas, Peru, 2017 and 2018. The sample universe was fifty-seven resolutions directors that resolve the prescription of PADs. The research methodology was of a basic type, descriptive, correlational, retrospective, and cross-sectional design. The instrument used was the check sheet. It was established that the largest $100 \%$ omission were two guarantees of due administrative procedure, which are: The right to the presumption of legality and compliance with reasonable terms. Likewise, the prescription of $98 \%$ of PADs occurred at the first moment for the initiation of PAD. Then it was concluded that the omission of due procedure by the administrative authorities did generate the prescription of disciplinary administrative procedures.
\end{abstract}

Keywords: Due procedure, prescription, disciplinary administrative procedure

${ }^{1}$ Bachiller en Derecho, Facultad de Derecho y Cencías Políticas de la Universidad Privada de Tacna. Correo electrónico: janine_cr@hotmail.com

${ }^{2}$ Docente asociado a Tiempo completo, Doctora en Ciencias de Enfermería de la Universidad Nacional Toribio Rodríguez de Mendoza de Amazonas. Correo electrónico: sonia.tejada@untrm.edu.pe

${ }_{3}^{3}$ Docente auxiliar a Tiempo completo, Magister. en Derecho Constitucional y Administrativo de la Universidad nacional de Trujillo. Correo electrónico: segundo.guevara@untrm.edu.pe 


\section{INTRODUCCIÓN}

La realidad de hoy nos muestra que las instituciones públicas del Estado en sus diferentes niveles, los servidores civiles en el desarrollo de sus labores y funciones, cometan faltas de carácter disciplinario desde leves hasta graves, como asistir al centro de trabajo en estado de embriaguez o bajo los efectos de las drogas, la negligencia en el desempeño de las funciones, la utilización o disposición de los bienes de la entidad pública en beneficio propio o de terceros. En ese sentido el Estado a través de la ley del servicio civil $\mathrm{N}^{\circ} .30057$ y su reglamento el D.S $\mathrm{N}^{\circ} 040-\mathrm{PCM}$, tienen como finalidad que las entidades públicas del estado alcancen mayores niveles de eficacia, eficiencia y calidad en la prestación de sus servicios. En consecuencia, es a través del procedimiento administrativo disciplinario (PAD) que se aplica la potestad sancionadora a los servidores civiles que infringen las normas afectando el adecuado funcionamiento de la administración pública, así como disuadir la comisión de faltas disciplinarias, y son las autoridades administrativas en quienes se centran la actuación y decisiones dentro de un debido procedimiento para que el resultado sea válido y justo. Sin embargo, los PAD lejos de concluir con una sanción, que puede ser una amonestación, suspensión, destitución o el archivamiento, estas terminan con la prescripción del PAD.

La naturaleza jurídica del debido proceso, se caracteriza como un derecho fundamental por excelencia del hombre como ser social y como deber de las autoridades administrativas que interactúan en el seno de la sociedad con las personas, estableciendo relaciones de convivencia que es reconocida y garantizada por nuestra Constitución Política del Perú que en el artículo 139 inc. 3, establece que: Ninguna persona puede ser desviada de la jurisdicción predeterminada por la ley, ni sometida a procedimiento distinto, de los previamente establecidos, ni juzgada por órganos jurisdiccionales de excepción ni por comisiones especiales creadas al efecto, cualquiera sea su denominación. Al respecto Hoyos, (1998), menciona que el debido proceso es un derecho fundamental complejo de carácter instrumental, que abarca numerosas garantías de las personas, y se considera como la mayor expresión del derecho procesal. Se trata de una institución jurídica que es parte integrante de la Constitución y que brinda la adhesión de los sujetos en busca de una tutela transparente de sus derechos. (p.54).

Mientras que la prescripción es aquella pérdida de la potestad sancionadora que faculta a las autoridades administrativas del PAD para investigar y sancionar al servidor civil que por inacción dejan que se venza el plazo de ley para resolver. Chámame (2015) refiere que la prescripción “(...) tiene dos modalidades de adquirir un derecho o librarse de una obligación por el transcurso del tiempo. Es la circunstancia que impide iniciar o continuar un proceso, así como de perder la facultad de penalizar a una persona, porque el término del plazo deja sin efecto el derecho de acción" (p.945). La ley del servicio civil ir señala que la prescripción del PAD se da en dos momentos, la primera antes del inicio del PAD y la segunda entre inicio del PAD y la resolución del órgano sancionador.

Dávila, (2017), señala que los motivos de la prescripción de los procesos administrativos disciplinarios son la falta de ética profesional, que comprende factores sociales, económicos, políticos, culturales agrega además a la corrupción, la injusticia, favores amistosos, comercio de influencias, desempeño en el trabajo y la transparencia en la información (p.77).

Bedón, (2016), muestra que las garantías del debido proceso como el derecho a ser escuchado, a la debida motivación y a recurrir consagradas en los literales c) i) y m) del numeral 7 del artículo 76 de la Constitución de la República del Ecuador, no se aplican efectivamente en el procedimiento disciplinario. (p. 106). Por otro lado, Surco, (2018), expresa que las normas del procedimiento sancionador del SAT vulneran el principio de proporcionalidad porque que el SAT no cumple con la valoración proporcional y adecuada de una infracción y que la ejecución coactiva vulnera el principio constitucional del debido proceso y que supletoriamente se aplica los principios del procedimiento sancionador de la Ley $\mathrm{N}^{\circ} 27444$, vulnerando el principio de legalidad (p.113).

\section{MATERIALY MÉTODO}

La investigación se realizó en la oficina de la Secretaría Técnica de la Dirección Regional de Salud (DIRESA) región Amazonas, Perú. El enfoque fue cuantitativo, diseño descriptivo, correlacional, retrospectivo y transversal (Páez, 2009, p.86). La población de estudio fue 150 denuncias de procedimientos administrativos disciplinarios (PAD) que ingresaron en el 2017 y 2018 en la Dirección Regional de Amazonas, 57 concluyeron con resolución directoral de prescripción. La muestra fue representada por 57 resoluciones directorales que resolvían la prescripción de los PAD. El muestreo aplicado fue no probabilístico por conveniencia.

Con autorización del Gobierno Regional de Amazonas y Dirección Regional de Salud de Amazonas, se realizó la observación indirecta de expedientes y resoluciones directorales, así mismo se otorgaron copias simples de las 57 resoluciones directorales que resolvieron la prescripción de las denuncias de los PAD de los años 2017 y 2018.

Se ubicó y seleccionó cada expediente por años 
conforme a la relación de ingresos de denuncias del PAD, en el sistema SISGEDO (Sistema de Gestión Documentaria) de la Dirección de Salud de Amazonas proporcionada por el secretario técnico de la DIRESA-Amazonas.

Se aplicó la técnica de observación indirecta, utilizando para ambas variables la ficha de cotejo, donde la primera parte correspondía al debido procedimiento administrativo, con una dimensión de garantías y derechos, y sus indicadores conforme a la guía sobre la aplicación del principio: derecho del debido proceso en los procedimientos administrativos (2013). La debida notificación, acceso al expediente, derecho a la defensa, presunción de licitud, a ofrecer y actuar pruebas, cumplimiento de los plazos razonablemente, investigación por autoridad competente, investigación por autoridad imparcial, impugnación de decisiones administrativas, $\mathrm{Ne}$ bis in ídem, principio de publicidad de normas procedimentales (p.10 al 29) donde sus índices fueron SI/NO

Respecto a la prescripción de los procedimientos administrativos disciplinarios se evaluó tres dimensiones: a) Por inacción de las autoridades competentes del PAD, con los indicadores, órgano instructor, órgano sancionador, jefe de recursos humanos, secretaria técnica y titular de la entidad, b) Por el tiempo transcurrido para servidores civiles, con los indicadores, para el inicio del PAD, a los tres años contados a partir de la falta, un año a partir de tomado conocimiento de la falta por la oficina de recursos humanos y un año entre el inicio del PAD y la emisión de la resolución administrativa, c) Por el tiempo transcurrido para ex servidores civiles, siendo el indicador a los dos años contados a partir de que la entidad conoció de la comisión de la infracción, con las opciones SI/NO.

\section{Tabla 1}

\section{RESULTADOS}

Frecuencia y porcentaje de omisión de las garantías del debido procedimiento administrativo en los PAD* durante los años 2017 y 2018.

\begin{tabular}{|c|c|c|c|c|c|c|}
\hline \multirow{3}{*}{$\begin{array}{c}\text { Garantías del debido procedimiento } \\
\text { administrativo }\end{array}$} & \multicolumn{4}{|c|}{ Omisión } & \multirow{3}{*}{$\begin{array}{c}\text { Total } \\
\text { f1 }\end{array}$} & \multirow{3}{*}{$\begin{array}{c}\text { Total } \\
\%\end{array}$} \\
\hline & \multicolumn{2}{|c|}{2017} & \multicolumn{2}{|c|}{2018} & & \\
\hline & f1 & $\%$ & f1 & $\%$ & & \\
\hline Debida notificación & 7 & 12 & 49 & 86 & 56 & 98 \\
\hline Acceso al expediente & 7 & 12 & 49 & 86 & 56 & 98 \\
\hline Derecho a la defensa & 7 & 12 & 49 & 86 & 56 & 98 \\
\hline Derecho a la presunción de licitud & 8 & 14 & 49 & 86 & 56 & 100 \\
\hline A ofrecer y producir pruebas & 7 & 12 & 49 & 86 & 56 & 98 \\
\hline Decisión motivada y fundada en derecho & 0 & 0 & 0 & 0 & 0 & 0 \\
\hline Cumplimiento de los plazos razonablemente & 8 & 14 & 49 & 86 & 56 & 100 \\
\hline Investigación por una autoridad competente & 1 & 2 & 0 & 0 & 0 & 2 \\
\hline Investigación por una autoridad imparcial & 0 & 0 & 0 & 0 & 0 & 0 \\
\hline Impugnación de decisiones administrativas & 0 & 0 & 0 & 0 & 0 & 0 \\
\hline Ne bis in ídem & 0 & 0 & 0 & 0 & 0 & 0 \\
\hline Principio de publicidad de normas procedimentales & 0 & 0 & 0 & 0 & 0 & 0 \\
\hline
\end{tabular}

*PAD: Procedimiento Administrativo Disciplinario.

En la tabla 1 se observa la mayor omisión del 100\% de dos garantías del debido procedimiento administrativo, que son: Derecho a la presunción de licitud y el cumplimiento de los plazos razonablemente, siendo la menor omisión correspondiente a la garantía: Investigación por autoridad competente, con un $2 \%$. 


\section{Tabla 2}

Frecuencia y porcentaje de las garantías del debido procedimiento administrativo infringidas en los PAD* en la fase de la actividad preparatoria durante los años 2017 y 2018.

\begin{tabular}{|c|c|c|c|c|c|c|}
\hline \multirow{3}{*}{$\begin{array}{c}\text { Garantías del debido procedimiento } \\
\text { administrativo infringidas en los PAD* en la fase } \\
\text { de la actividad preparatoria }\end{array}$} & \multicolumn{4}{|c|}{ Año } & \multirow{3}{*}{$\begin{array}{c}\text { Total } \\
\text { f1 }\end{array}$} & \multirow{3}{*}{$\begin{array}{c}\text { Total } \\
\%\end{array}$} \\
\hline & \multicolumn{2}{|c|}{2017} & \multicolumn{2}{|c|}{2018} & & \\
\hline & f1 & $\%$ & f1 & $\%$ & & \\
\hline Derecho a la notificación al denunciante & 7 & 12 & 49 & 86 & 56 & 98 \\
\hline Acceso al expediente & 7 & 12 & 49 & 86 & 56 & 98 \\
\hline Derecho a la defensa de la entidad & 7 & 12 & 49 & 86 & 56 & 98 \\
\hline Actuación de las pruebas de la entidad & 7 & 12 & 49 & 86 & 56 & 98 \\
\hline Derecho a la presunción de licitud & 7 & 12 & 49 & 86 & 56 & 98 \\
\hline $\begin{array}{l}\text { Tramitación de la denuncia y diligencias dentro del } \\
\text { plazo razonable }\end{array}$ & 7 & 12 & 49 & 86 & 56 & 98 \\
\hline
\end{tabular}

*PAD: Procedimiento Administrativo Disciplinario.

En la tabla 2 se muestra que seis garantías del debido procedimiento administrativo han sido infringidas en la fase de la actividad preparatoria en los PAD con un 98\% cada una, existiendo un incremento significativo del año 2017 al 2018 con un valor de $12 \%$ y $86 \%$, respectivamente.

\section{Tabla 3}

Frecuencia y porcentaje de las garantías del debido procedimiento administrativo infringidas en los PAD* en la fase instructiva durante el año 2017.

\begin{tabular}{lcc}
\hline \multicolumn{1}{c}{$\begin{array}{c}\text { Garantías del debido procedimiento administrativo infringidas en los } \\
\text { PAD* en la fase instructiva }\end{array}$} & \multicolumn{1}{c}{ Año } \\
\cline { 2 - 3 } & & $\mathbf{2 0 1 7}$ \\
\hline Derecho a la presunción de licitud & 1 & $\mathbf{f}$ \\
Cumplimiento de los plazos razonablemente & 1 & 2 \\
Investigación por autoridad competente & 1 & 2 \\
\hline
\end{tabular}

*PAD: Procedimiento Administrativo Disciplinario.

En la tabla 3 se observa que tres garantías del debido procedimiento administrativo han sido infringidas en los PAD durante la fase instructiva con un $2 \%$ cada una.

\section{Tabla 4}

Frecuencia y porcentaje de extinción de la potestad de investigar y sancionar en las fases del PAD* durante los años 2017 y 2018 .

\begin{tabular}{|c|c|c|c|c|c|c|c|}
\hline \multirow{3}{*}{$\begin{array}{l}\text { Extinción de la potestad de investigar } \\
\text { las fases del PAD }\end{array}$} & \multirow{3}{*}{ y sancionar en } & \multicolumn{4}{|c|}{ Año } & \multirow{3}{*}{$\begin{array}{c}\text { f1 } \\
\text { total }\end{array}$} & \multirow{3}{*}{$\begin{array}{c}\% \\
\text { total }\end{array}$} \\
\hline & & \multicolumn{2}{|c|}{2017} & \multicolumn{2}{|c|}{2018} & & \\
\hline & & f1 & $\%$ & f1 & $\%$ & & \\
\hline Fase de actividad preparatoria & & 7 & 12 & 49 & 86 & 56 & 98 \\
\hline Fase instructiva & & 1 & 2 & 0 & 0 & 1 & 2 \\
\hline Fase sancionadora & & 0 & 0 & 0 & 0 & 0 & 0 \\
\hline Total & & & & & & 57 & $100 \%$ \\
\hline
\end{tabular}

*PAD: Procedimiento Administrativo Disciplinario.

En la tabla 4, se demuestra que en un 98\% (56) de las resoluciones directorales se extinguió la potestad de investigar en la fase de la actividad preparatoria del PAD. Mientras que en un 2\% (1) se extinguió la potestad de investigar en la fase instructiva y la potestad de sancionar en un $0 \%$ en la fase sancionadora. 


\section{Tabla 5}

Frecuencia y porcentaje del momento en que se produjo la prescripción de los procedimientos administrativos disciplinarios durante los años 2017 y 2018.

\begin{tabular}{|c|c|c|c|c|c|}
\hline \multirow{3}{*}{ Momento en que se produjo la prescripción del PAD } & \multicolumn{4}{|c|}{ Año } & \multirow{3}{*}{$\begin{array}{c}\% \\
\text { total }\end{array}$} \\
\hline & \multicolumn{2}{|c|}{2017} & \multicolumn{2}{|c|}{2018} & \\
\hline & f1 & $\%$ & f1 & $\%$ & \\
\hline Para el inicio del PAD* & 7 & 12 & 49 & 88 & 98 \\
\hline Entre el inicio del PAD $*$ y el termino & 1 & 2 & 0 & 0 & 2 \\
\hline
\end{tabular}

*PAD: Procedimiento Administrativo Disciplinario.

En la tabla 5 se observa que el $98 \%$ de las prescripciones se dieron en el momento en que se inicia el procedimiento administrativo disciplinario durante los años 2017 y 2018.

\section{DISCUSIÓN}

Los resultados de esta investigación han demostrado que existe una omisión del 100\% de dos garantías del debido procedimiento del (PAD) que son, el derecho a la presunción de licitud y el cumplimiento de los plazos razonablemente y siendo la menor omisión correspondiente a la garantía: Investigación por autoridad competente, con un $2 \%$. (tabla 1 ). Coincidimos en parte con los resultados encontrados por Román (2014) cuando afirma que, en el plano procedimental, la Administración, en el ejercicio de estas potestades, deberá observar el derecho a la defensa jurídica, a la presunción de inocencia y al acceso a la justicia (p.204). Como se advierte nuestro resultado coincide cuando considera que en los procesos administrativos debe observarse la garantía del derecho a la presunción de inocencia o de licitud. Al respecto Peña (2010) define que la omisión tiene como elementos: La inactividad o abstención voluntaria, relación de causalidad y resultado antijurídico, consecuencia del comportamiento omisivo (p. 115). Así mismo coincidimos con Morón (2017) cuando menciona que "Los administrados tienen el derecho a la no desviación del procedimiento administrativo" (p.78).

En la (tabla 2) se demostró que un 98\% (56) en cada una de las resoluciones directorales, se llegó a identificar a seis garantías infringidas del debido procedimiento en la fase de la actividad preparatoria de los PAD, siendo el derecho a la notificación al denunciante, el acceso al expediente, derecho a la defensa de la entidad, la actuación de las pruebas de la entidad, derecho a la presunción de licitud, tramitación de la denuncia y diligencias dentro del plazo razonable. Así mismo se advierte un incremento significativo del año 2017 al 2018 con un valor de $12 \%$ y $86 \%$, respectivamente. Mientras que en la (tabla 3) se demostró tres garantías del debido procedimiento administrativo, que se han infringido durante la fase instructiva con un $2 \%$ cada una, con el derecho a la presunción de licitud, cumplimiento de los plazos razonablemente, investigación por autoridad competente. Al respecto coincidimos en parte con los resultados de Bedon (2016) cuando identifica las garantías del debido procedimiento que se vulneraron, como el derecho a ser escuchado, a la debida motivación, como inaplicables en el procedimiento disciplinario y que en los fallos los argumentos carecen de coherencia entre los hechos, las normas y las pruebas. Como se advierte en la presente investigación se ha identificado como una de las garantías infringidas en los PAD el derecho a la defensa dentro de la cual se encuentra inmersa el derecho a ser escuchado. También coincidimos en parte con los resultados por Surco, (2018). Cuando afirma que en el procedimiento sancionador del SAT al ejecutar cobros por infracciones de papeletas de tránsito se vulnera el principio del debido procedimiento al no motivar debidamente las notificaciones conforme al $60 \%$ de encuestados (p.113).

Los resultados de la (tabla 4) demostraron que en un $98 \%$ (56) de las resoluciones directorales se extinguió la potestad de investigar en la fase de la actividad preparatoria del PAD. Mientras que en un $2 \%$ (1) se extinguió la potestad de investigar en la fase instructiva y la potestad de sancionar en un $0 \%$ en la fase sancionadora. Tales resultados difieren con Bartra (2017), cuando refiere que la competencia para resolver un PAD recae en un mismo órgano instructor o sancionador, esta pierde objetividad e imparcialidad. Así mismo coincidimos en parte con los resultados de Huamán (2019) cuando afirma que las sanciones disciplinarias impuestas al servidor del Hospital de Chancay que Trabaja al servicio del Estado en el 2018, dicho proceso presentan vicios de Nulidad, a pesar de todo la Secretaria Técnica, fuerza groseramente el acto administrativo vulnerando la Ley del Servicio Civil "Ley 30057", se vulnera el Principio de Debido Procedimiento previsto en el numeral 2 de la Ley 27444, Principio de tipicidad 
previsto en el numeral 4 del $\operatorname{Art}^{\circ} 230$ de la Ley 27444 , Principio de Presunción de Inocencia previsto en el numeral 9 del $\mathrm{Art}^{\circ} 230$ de la Ley 27444.

Por último, los resultados de la (tabla 5) demuestran que del $100 \%$ de resoluciones directorales en el 98\% se dieron las prescripciones en el momento en que se inicia el procedimiento administrativo disciplinario durante los años 2017 y 2018. Entonces si la prescripción promueve que la autoridad administrativa pierda competencia para investigar o sancionar a un servidor civil que ha cometido una falta disciplinaria administrativa coincidimos con lo resuelto por la Sala Plena del Tribunal del Servicio Civil (2016) señala que “(...) la prescripción es una forma de liberar a los administrados de las responsabilidades disciplinarias que les pudiera corresponder, originada por la inacción de la Administración Pública, quien implícitamente renuncia al ejercicio de su poder sancionador (fs.21). Existe similitud con el estudio de Delgado (2019) en Cajamarca, Perú; quién afirmó que no se ha cumplido en su totalidad el proceso del debido procedimiento en la aplicación de sanciones administrativas disciplinarias en la Ley del Servicio Civil en la Red de Salud. Los resultados de Dávila (2017) difieren con lo que aquí se encontró; es decir que la causa de la prescripción, es la falta de ética profesional por factores sociales, económicos, políticos, cultural, desinterés de los encargados competentes, favores amicales, injusticia, impunidad y desconocimiento de las consecuencias jurídicas administrativas

\section{CONCLUSIONES}

Existe la omisión del $100 \%$ de dos garantías del debido procedimiento administrativo disciplinario (PAD), que son: Derecho a la presunción de licitud y el cumplimiento de los plazos razonablemente, siendo la menor omisión correspondiente a la garantía: Investigación por autoridad competente, con un $2 \%$

Seis garantías del debido procedimiento administrativo disciplinario han sido infringidas en los PAD con un $98 \%$ cada una, existiendo un incremento significativo del año 2017 al 2018 con un valor de $12 \%$ y $86 \%$, respectivamente.

Tres garantías del debido procedimiento administrativo han sido infringidas en los PAD durante la fase instructiva con un $2 \%$ cada una.

El 98\% (56) de las resoluciones directorales se extinguió la potestad de investigar en la fase de la actividad preparatoria del PAD. Mientras que en un $2 \%$ (1) se extinguió la potestad de investigar en la fase instructiva y la potestad de sancionar en un $0 \%$ en la fase sancionadora.

El 98\% (56) de las prescripciones de los PAD se dieron en el momento en que se inicia el procedimiento administrativo disciplinario durante los años 2017 y 2018.

\section{REFERENCIAS BIBLIOGRÁFICAS}

Bartra, E. (2017). La afectación del debido procedimiento por la aplicación del régimen de competencia disciplinaria previsto en la ley del servicio civil. (Tesis de maestría). Universidad San Martin de Porras. Lima Perú.

Bedón, N. (2016). El debido proceso en los procedimientos administrativos para el ejercicio de la potestad disciplinaria del Consejo de la Judicatura. (Tesis de pregrado). Universidad de las Américas. Quito-Ecuador.

Constitución Política del Perú. Art 139, inc., 3. (1993). Lima- Peru: Edit. Ediciones Legales E.I.R.L

Chanamé, Capítulo VIII - Poder Judicial (Art. 138 al 149)," en: La Constitución comentada, vol. 2,(Lima: Ediciones Legales, 2015), p. 945.

Delgado, S. (2020). Gestión de los procesos administrativos disciplinarios en la ley del servicio civil en la red de salud cutervo, 2019. https://repositorio.utelesup.edu.pe/handle/ut elesup/1069

Dávila, Y. (2017). “Conducta ética como causal de prescripción de los procesos administrativos disciplinarios en la Gerencia Regional de Salud Lambayeque -2012-2014". (Tesis de maestría). Universidad Cesar Vallejo. Chiclayo-Perú.

Ministerio de Justicia y Derechos Humanos (2013). Guía sobre la aplicación del principio derecho del debido proceso en los procedimientos administrativos ( $1^{\mathrm{a}}$ ed.). Lima, Perú: Distribución gratuita.

Hoyos, (1998). El Debido Proceso, ColombiaBogotá: Temis.

Huamán, E (2019). Irregularidades en el desarrollo de los procedimientos administrativos disciplinarios por parte del Hospital de Chancay en el marco de la Ley Servir y su Reglamento, 2018. (Tesis de pregrado). Universidad Privada Telesup. Lima-Perú

Ley del Servicio Civil- Ley N ${ }^{\circ}$ 30057-2013. https://leyes.congreso.gob.pe/Documentos/ Leyes/30057.pdf 
Morón, U. (2017). Comentarios a la Ley del Procedimiento Administrativo General Tomo II (12 ${ }^{a}$ ed.). Lima, Perú: Gaceta Jurídica.

Páez, J. (2009). El plan de tesis, impresiones Olograf. Estudios y Ediciones R.A

Peña, O. (2010). Teoría del Delito: manual práctico para su aplicación en la teoría del caso. $\left(1^{\mathrm{a}}\right.$ ed.). Lima, Perú: Edit. Nomos \& Thesis E.I.R.L.

Reglamento el D.S N $\mathrm{N}^{\circ}$ 040-2014 http://www. minedu.gob.pe/politicas/pdf/ley-30057/dsn040-2014-pcm.pdf

Resolución de Sala Plena No 001-2016 SERVIR/TSC. (2016). Precedente Administrativo de observancia obligatoria para determinar la correcta aplicación de las normas que regulan la prescripción de la potestad disciplinaria en el marco de la ley $N^{\circ} 30057$ y su reglamento. Diario oficial del Bicentenario - El Peruano.

Román, C (2014). El debido procedimiento administrativo sancionador. Revista de Derecho Público, (71), Págs. 183-214. doi:10.5354/0719-5249.2014.35692

Santos Moreira, M. P. (2021). Las sanciones administrativas establecidas en la ley orgánica de salud y la desproporcionalidad al momento de resolver (Master's Thesis, Guayaquil: ULVR, 2021.).

Surco, M (2018). El procedimiento administrativo sancionador y la vulneración de los principios constitucionales (Tesis de pregrado). Universidad Autónoma del Perú. Lima-Perú. (67) 177-188.

Zelayaran, M. (1997). Metodología de investigación jurídica. Lima, Perú: Edit. Ediciones Jurídicas. 